\title{
Probing the Active Sites of ZnO Nanowire Supported Ir Species for CO Oxidation
}

Jia Xu${ }^{1}$, Yafeng $\mathrm{Cai}^{1,2}, \mathrm{Xu} \mathrm{Li}^{1,3}$ and Jingyue $\mathrm{Liu}^{1 *}$

1. Department of Physics, Arizona State University, Tempe, Arizona 85287, United States

2. Key Laboratory for Advanced Materials and Research Institute of Industrial Catalysis, East China University of Science and Technology, Shanghai 200237, China

${ }^{3}$ Hefei National Laboratory for Physical Sciences at the Microscale, University of Science and Technology of China, Hefei, Anhui 230026, China

* Corresponding author: Jingyue.liu@asu.edu

The atomicity and configuration of metal nanoclusters play an important role in controlling their adsorption and catalytic behaviors. The recent rapid development of single-atom catalysis [1-2] has attracted intense interest in understanding heterogeneous catalysis by supported metal clusters [3]. For metal clusters with sizes in the subnanometer range, not only the total number of atoms but also the specific geometric arrangement of the individual atoms within the cluster and their interactions with the support surfaces become critical in controlling their reactivity. We have previously reported that under the appropriate gas treatment $\mathrm{ZnO}$ nanowire (NW) supported Ir clusters can be transformed into single-atom chains and furthermore the Ir atom chains can oxidize $\mathrm{CO}$ at moderate temperatures [4]. In this work, we developed a method to reversibly transform Ir single-atom chains to highly disordered subnanometer Ir clusters or well-crystallized Ir clusters. We further used $\mathrm{CO}$ oxidation as a probe to evaluate the catalytic properties of the different configurations of the ZnO NW supported Ir clusters. The correlation between cluster structure and catalytic performances demonstrates that well-crystallized clusters possess the highest activity for $\mathrm{CO}$ oxidation.

The Ir /ZnO sample was synthesized by a modified strong adsorption method. Briefly, Ir metal precursors were dropwise added into the solution of pre-formed $\mathrm{ZnO}$ NWs. The resultant precipitate was filtered, washed with deionized water and dried. The dried $\mathrm{Ir} / \mathrm{ZnO}$ NWs were then calcined in air at $300^{\circ} \mathrm{C}-500^{\circ} \mathrm{C}$ for 2-10 hrs to form single-atom Ir chains on $\mathrm{ZnO}$ NWs [4]. Post-treatment in $\mathrm{CO}$ and $\mathrm{H}_{2}$ was carried out to transform the single-atom Ir chains into atomically dispersed, sub-nanometer Ir clusters (under 10 atoms) or well-crystallized Ir crystallites with an average size of $\sim 1.3 \mathrm{~nm}$. The Aberration-corrected HAADF-STEM, indispensable for investigating the active species in supported metal atom and cluster catalysts [5], was used to examine the self-assembled Ir single-atom chains, the highly disordered Ir clusters and the well-crystallized Ir nanoclusters.

Three pairs of low and high magnification STEM-HAADF images are shown in Figure 1 (a-b: Ir singleatom chains; c-d: Ir sub-nanometer clusters; and e-f: well-crystallized Ir clusters). The Ir single-atom chains preferentially grow on the $\mathrm{ZnO}\{10-10\}$ nanofacets (marked by the yellow dotted lines in Figure1ab) [4]. After treating the supported single-atom $\mathrm{Ir}$ chains in $\mathrm{CO}$ and $\mathrm{H}_{2}$ at the selected temperatures, we transformed the single-atom Ir chains into subnanometer clusters (dimers, trimers, etc.) with various sizes and configurations including highly disordered sub-nanometer Ir clusters (indicated by the red circles in Figure 1c-d). Under a different post-treatment condition, the single-atom Ir chains were transformed into well-crystallized Ir clusters (indicated by the blue arrows in Figurele-f). These Ir crystallites are strongly anchored onto the $\mathrm{ZnO}\{10-10\}$ nanofacets, probably by an epitaxial relationship. Since the various configurations of the Ir clusters can be in situ transformed in a catalytic reactor we have conducted CO oxidation reaction on the same $\mathrm{ZnO}$ supported Ir catalyst but with different types of Ir configurations that 
were accomplished by in situ gas treatment (Figure 1g). The experimental data unambiguously showed that the activity of $\mathrm{CO}$ oxidation depends on the atomicity and cluster structure: well-crystallized Ir clusters (blue plot) $>>$ highly disordered subnano Ir clusters (red plot) >> Ir single-atom chains (black plot). This result demonstrates that the coordination number and bonding of Ir atoms as well as their interactions with the $\mathrm{ZnO}\{10-10\}$ surfaces may play an important role in determining their catalytic activity for $\mathrm{CO}$ oxidation [6].

\section{References:}

[1] B Qiao et al., Nature Chemistry 3 (2011), p. 634.

[2] JY Liu, ACS Catalysis 7 (2017), p. 34.

[3] EC Tyo and S Vajda, Nature Nanotechnology 10 (2015), p. 577.

[4] J Xu et al, Microscopy and Microanalysis 23. S1 (2017) p.484.

[5] JY Liu, Chinese Journal of Catalysis 38 (2017), p. 1460.

[6] This work was funded by NSF under CHE-1465057. We acknowledge the Eyring Materials Center and the John M. Cowley Center for High Resolution Electron Microscopy at Arizona State University.
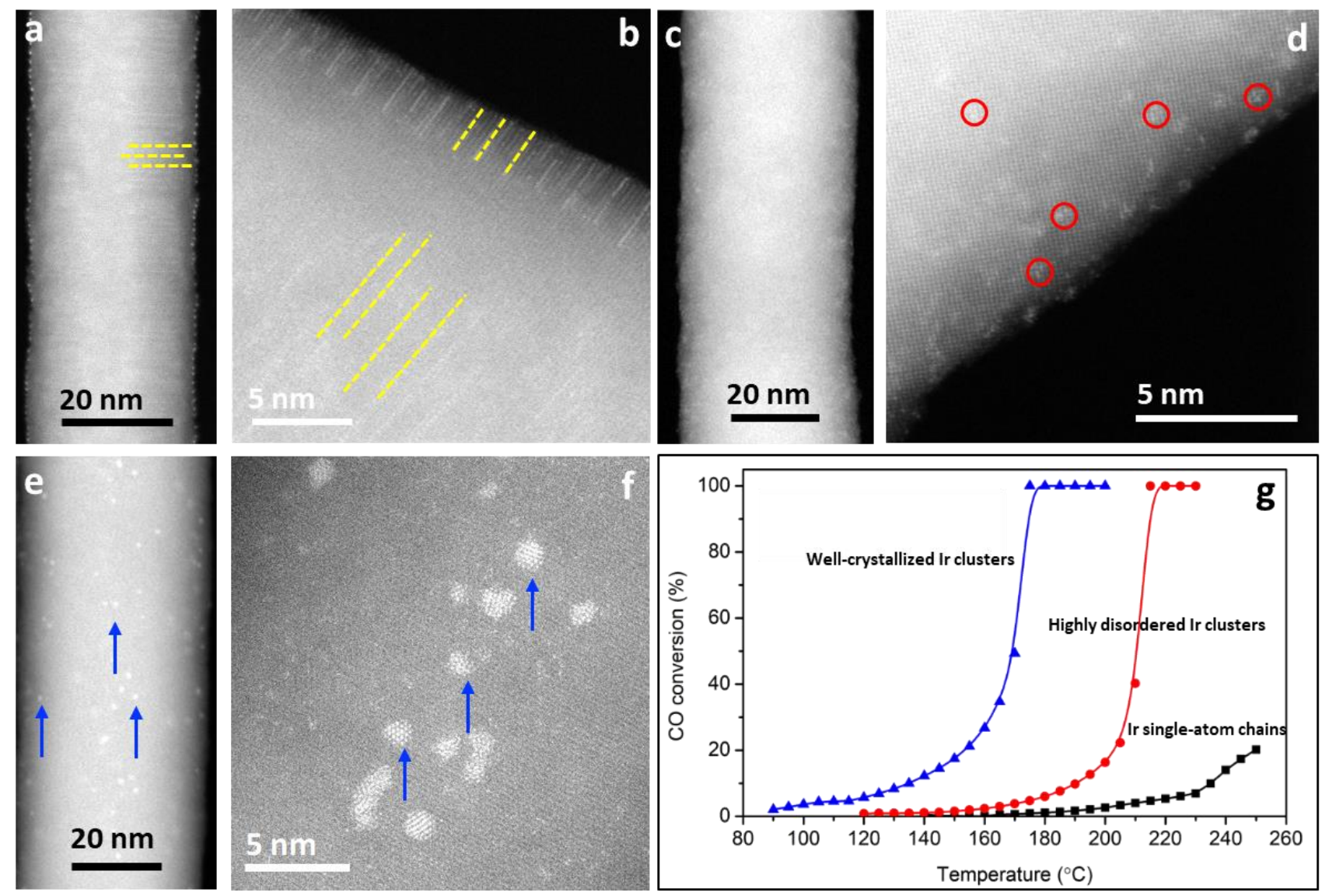

Figure 1. HAADF images of $\mathrm{ZnO}$ supported: (a-b) single-atom $\mathrm{Ir}$ chains/ZnO $\mathrm{NW}$, (c-d) highly disordered subnanometer Ir clusters and (e-f) well-crystallized Ir clusters. The yellow dotted lines, red circles and blue arrows indicate single-atom Ir chains, highly disordered small Ir clusters and wellcrystallized Ir clusters. Figure 1g shows the conversion rates for $\mathrm{CO}$ oxidation on the corresponding catalysts. Reaction condition: $1 \mathrm{vol} \% \mathrm{CO}+4 \mathrm{vol} \% \mathrm{O}_{2}$ and balance with $\mathrm{He}$, WHSV $=10,000 \mathrm{mlg}^{-1} \mathrm{~h}^{-1}$. 